\title{
EchoGéo
}

44 | 2018

Les valorisations territoriales et touristiques du street art

\section{Le productivisme au travail : paroles d'agriculteurs beaucerons}

Entretien avec Gatien Elie, réalisé à Paris, le 22 mai 2018

\section{Gatien Elie and Serge Weber}

\section{OpenEdition}

\section{Journals}

Electronic version

URL: https://journals.openedition.org/echogeo/15373

DOI: 10.4000/echogeo.15373

ISSN: 1963-1197

Publisher

Pôle de recherche pour l'organisation et la diffusion de l'information géographique (CNRS UMR 8586)

\section{Electronic reference}

Gatien Elie and Serge Weber, "Le productivisme au travail : paroles d'agriculteurs beaucerons", EchoGéo [Online], 44 | 2018, Online since 31 July 2018, connection on 31 July 2021. URL: http:// journals.openedition.org/echogeo/15373 ; DOI: https://doi.org/10.4000/echogeo.15373

This text was automatically generated on 31 July 2021.

EchoGéo est mis à disposition selon les termes de la licence Creative Commons Attribution - Pas d'Utilisation Commerciale - Pas de Modification 4.0 International (CC BY-NC-ND) 


\title{
Le productivisme au travail : paroles d'agriculteurs beaucerons
}

\author{
Entretien avec Gatien Elie, réalisé à Paris, le 22 mai 2018
}

\section{Gatien Elie and Serge Weber}

1 Au sein d'une toute jeune collection, L'ordinaire du capital, les éditions Amsterdam ont fait paraitre en 2017 quatre ouvrages qui ne peuvent qu'attirer l'attention des lecteurs géographes. Le propriétaire absent, de Takiji Kobayashi, un classique de la littérature prolétarienne japonais, est une chronique des luttes paysannes à Hokkaido dans les années vingt. Les briques rouges, de Quentin Ravelli, biographie sociale de marchandise, nous plonge dans l'industrie de la brique, des carrières d'argile jusqu'aux villes fantômes de la Sagra, une région pauvre d'Espagne exposée à la crise financière. Enfin, La petite ville d'Éric Chauvier et La Plaine de Gatien Élie explorent, chacun à leur façon, les conditions actuelles, peu médiatisées, de la ruralité en France.

Dans La Plaine, le géographe Gatien Elie nous livre une passionnante ethnographie du travail dans un des espaces ruraux les plus emblématiques des " grandes agricultures » françaises, la Beauce. Son investigation explore le monde social et économique des exploitants agricoles. Ces derniers expriment, au gré de portraits et de situations, leur point de vue sur les injonctions contradictoires auxquelles ils doivent faire face chaque jour, dont les deux principaux pôles sont la dépendance au marché et l'exposition récurrente aux produits phytosanitaires toxiques. Ces portraits saisissants, qui nous montrent leurs manières de "faire avec", sont éclairés par une mise en miroir de facteurs explicatifs, insérés avec autant d'efficacité que de discrétion, un subtil dosage que rend possible un choix d'écriture scientifique original.

3 - Serge Weber (SW). Je voudrais tout d'abord vous inviter à revenir sur l'écriture en tant que telle. Vous avez fait un choix très original pour inclure la parole recueillie dans l'énonciation, dans le corps même d'une narration à la fois distanciée et très empathique, qui ménage une réelle place à l'autre. Le discours est en effet à mi-chemin entre discours indirect et indirect libre, au gré de chapitres courts, alternant portraits et analyses. 
- Gatien Elie (GE). Durant les trois ans qu'a duré ce terrain, j'ai effectué des séjours entrecoupés, pendant les vacances scolaires. Et au fur et à mesure que le livre avançait, je me suis rendu dans la Beauce de plus en plus souvent et de plus en plus longtemps. Durant la dernière phase d'écriture, entre le printemps et l'automne 2017, j'y allais régulièrement, parfois même pour un seul entretien. Je prenais alors le temps de rouler, à la recherche de lieux à photographier pour nourrir mes descriptions de paysage. J'ai commencé à écrire un an après mes premières enquêtes de terrain. Des bribes dans un premier temps. Allan Popelard, mon éditeur et directeur de la collection, me disait si j'allais dans la bonne direction et me donnait des idées d'écriture. Nous avions par exemple imaginé une forme théâtrale, le livre aurait pu devenir une grande pièce de théatre.

- SW. Gageons qu'il sera un jour adapté pour la scène, il s'y prête plutôt bien !

6 - GE. J'ai passé beaucoup de temps avec les exploitants, le plus souvent la matinée entière, suivie d'un déjeuner, et j'en sortais avec quatre heures d'enregistrement que je retranscrivais intégralement. J'ai essayé d'identifier des idéaltypes selon la dimension du productivisme que chacun des agriculteurs permettait d'interroger. J'ai donc fait le choix de construire le livre autour de portraits. Pour passer de blocs de parole brute à une forme littéraire, il a fallu de nombreux va-et-vient avec l'éditeur. Nous avons fait plusieurs tentatives pour trouver une énonciation satisfaisante. Nous aurions pu tout laisser à la première personne, mais cela supposait une écriture très contraignante : cela m'empêchait de ré-agencer les phrases, d'inclure des informations. En effet, dans les portraits, il y a toujours cette double lecture : ce qui est dit par l'agriculteur et les informations de contextualisation nécessaires pour le comprendre. Choisir au contraire la troisième personne permettait une écriture plus libre, plus fluide à la lecture. Ces entretiens ont été considérablement condensés, nous sommes passés d'environ quinze pages à deux ou trois pages chacun. J'ai gardé ce qui était le plus touchant, le plus sensible, et à la fois le plus problématique.

7 - SW. Finalement, pourquoi avoir choisi des portraits?

8 - GE. C'est une vieille question... Quand j'ai soutenu mon Master 1 avec Georgette Zrinscak, il y avait également dans le jury Béatrice Collignon. J'avais fait un terrain sur la pauvreté en milieu rural dans l'Hérault, avec beaucoup d'enquêtes. On m'a reproché de ne pas laisser assez de place aux enquêtés, je prenais la parole à leur place, on ne saisissait pas assez leur vie, leur trajectoire, leur vision du monde et de leur territoire. En évoquant un jour l'idée des portraits, Allan venait ainsi valider une forme à laquelle j'avais depuis longtemps réfléchi.

9 - SW. Ce qu'on en ressent à la lecture est une impression de profondeur, de proximité, une place faite à l'accueil : vous évitez les positions de surplomb, vous vous gardez bien de toute trace d'interprétation sauvage et vous êtes particulièrement économe en parapluies théoriques qui auraient semblé déplacés dans cette démarche accomplie de décentrement. J'ai été particulièrement sensible à cette proposition méthodologique très rigoureuse, à la lisière de l'écriture littéraire et de l'écriture scientifique, qui apporte un écho très pertinent aux questionnements actuels sur les savoirs situés et la réflexivité des chercheurs, qui est devenue un préalable indispensable à toute production scientifique de qualité. Pourriez-vous revenir sur les lectures qui vous ont inspiré, qui vous rattachent à certaines filiations d'écriture et de recherche? 
10 - GE. Parmi les livres qui m'ont nourri, il y a les témoignages de Robert Linhart, Le Sucre et la Faim (1981) et L'Établi (1978) que j'aime énormément, en particulier parce qu'ils mettent en scène les discussions des travailleurs. De même, L'Eil écoute de Claudel (1946), qui décrit très bien les paysages des Flandres, les grands ciels, cette plaine. Je suis sensible à diverses manières de décrire la campagne, par exemple celle de Bernard Charbonneau, dans Tristes campagnes (1973), qui a une façon très belle de faire attention aux menus détails révélateurs de profondes transformations. Mais aussi celles des Géographies universelles du vingtième siècle : dans les Larousse (1960) ou les Quillet (1928), les paysages ruraux sont décrits avec une certaine dramaturgie: l'anthropisation est toujours une lutte contre les forces géomorphologiques et les vicissitudes du climat. Pour le contenu scientifique, les influences sont nombreuses, à commencer par l'histoire des sciences et des techniques portée par un groupe autour de François Jarrige (2009), Jean-Baptiste Fressoz (2012), Christophe Bonneuil (2012; 2013), Nathalie Jas (2005). Tous ces historiens ou sociologues ont en commun de faire une histoire critique des sciences, une autre histoire du progrès. Ils avaient organisé en 2011, à l'EHESS, le colloque Une autre histoire des Trente glorieuses, interrogeant la mécanisation, la chimisation et plus généralement le progrès... Cette littérature m'a beaucoup inspiré, notamment lorsque j'interrogeais des semenciers ou des vendeurs de machines. Cela m'a permis d'historiciser et de relativiser la notion de progrès, d'apporter du contenu, par exemple autour de cette notion de " pureté de la semence » (Bonneuil, 2013, op. cit.). Ensuite, sur le thème du travail en général, je suis très redevable aux travaux de psychopathologie de Christophe Dejours (1998), qui éclaire tellement de choses... J'étais précisément en train de lire Dejours lorsque j'ai assisté à la réunion pour la formation à l'usage des produits phytosanitaires. J'avais sous les yeux un exemple emblématique de ce dont traite Dejours : un moment de neutralisation du risque professionnel, de banalisation de la violence au travail. C'était concordant avec tous les mécanismes qu'il décrit dans d'autres contextes : des démonstrations de virilité face au danger, d'euphémisation des risques par l'utilisation d'un langage technique et de procédures conformes aux réglementations, des moments d'humour et de dérision. Tout concourt à faire en sorte qu'ils acceptent de manipuler des produits toxiques. Ce n'est pas évident d'entrer dans un univers professionnel aussi complexe : il y a un vocabulaire propre, des manières de penser, des connivences, des blagues. Si je n'avais pas lu Dejours à ce moment, je serais passé à côté de ce qui se jouait. Les travaux sur le syndicalisme agricole aussi m'ont été nécessaires. Je viens moi-même du syndicalisme mais la différence est grande entre les syndicats de salariés et ceux de chefs d'exploitation agricoles. Il m'a fallu entrer dans l'histoire de ces organisations pour comprendre l'écart avec mon expérience personnelle. Par exemple, les maladies professionnelles sont une question à part entière dans mon syndicat mais pas du tout à la Fédération nationale des syndicats d'exploitants agricoles (FNSEA) ou à la Coordination rurale. À chaque fois que j'interrogeais leurs cadres, ces derniers bottaient en touche: ils voyaient les produits toxiques comme de simples outils de travail assortis d'une réglementation à respecter. Et pour cause, ce sont les syndicats et leurs coopératives qui, pour défendre les intérêts économiques de la profession, ont depuis le $\mathrm{XIX}^{\mathrm{e}}$ siècle introduit dans les campagnes toutes les innovations techniques, chimie de synthèse comprise...

11 - SW. Peut-être pourriez-vous préciser les différents points de vue des agriculteurs, dont on comprend dans le livre qu'ils peuvent diverger sensiblement. 
12 - GE. L'idée de départ, le synopsis, c'était de faire le tour de tous les métiers qui gravitent autour du chef d'exploitation, le banquier, le formateur, etc., et de voir les responsabilités des uns et des autres dans le système. Il en manque cependant dans le livre : par exemple, on ne voit pas apparaître d'enseignants des lycées professionnels agricoles qui sont pourtant des acteurs importants. J'ai rencontré des personnes qui travaillaient dans ce milieu, mais je n'avais pas assez de matière pour en faire état. Il manque également la voix des industriels produisant les phytosanitaires: Bayer, Monsanto, Singenta, BASF... Si je ne les ai pas rencontré, c'est parce qu'ils ne sont pas présents dans la plaine. Ils ont des représentants commerciaux. De plus, une bonne partie des produits sont encore fournis directement aux exploitants par les coopératives. Il manque aussi les contrôleurs, ceux de l'Union européenne ou ceux du ministère de l'Agriculture. J'aurais aimé assister à un contrôle, mais l'occasion ne s'est pas présentée. Pour le reste, le livre présente à mon sens une diversité d'acteurs intéressante. Pour chacun des acteurs, j'ai essayé d'entrer dans leur logique. Non pas d'en faire des marchands de mort ou des coupables, mais d'entrer dans leur rationalité et dans leurs contraintes, dans ce qui fait leur métier. Aux yeux du banquier, par exemple, la dette des chefs d'exploitation n'est pas un problème mais un outil pour aider l'agriculteur à sortir d'une mauvaise passe. S'il n'y avait pas eu ce recours au crédit qui a permis aux exploitants de se maintenir, il n'y aurait aujourd'hui, selon lui, plus que quelques immenses exploitations de plusieurs milliers d'hectares, qui appartiendraient à de grands investisseurs. De même pour la formatrice de la Chambre d'agriculture: elle met en garde les agriculteurs sur les risques liés aux produits phytosanitaires - qui sont détaillés explicitement - tout en indiquant les «bonnes pratiques » sensées prémunir contre ces risques. Et de ce point de vue, elle fait très bien son travail.

13 - SW. Pourriez-vous justement revenir sur ces produits toxiques, qui constituent le cœur de ce qu'on pourrait appeler l'intrigue sous-jacente du livre?

GE. Historiquement, la chimie de synthèse a permis d'accroitre les rendements, en détruisant les insectes « nuisibles » et les maladies. Aujourd'hui, si les agriculteurs de la Beauce se passaient de la chimie de synthèse, leurs rendements s'effondreraient. De manière générale tous les agriculteurs qui passent en bio voient dans un premier temps leur production baisser car il faut réorganiser toute l'exploitation, repenser différemment la protection des plantes. Pour les agriculteurs que j'ai rencontrés, une chute de rendements - même circonscrite dans le temps - signifie une chute de revenu inacceptable. Il y a un blocage économique évident, et l'annonce récente du Ministère ne va rien arranger : des aides à la conversion en bio existent toujours mais les « aides au maintien » ne seront pas versées cette année, faute de moyens. Mais il y a aussi un blocage idéologique, bien qu'il soit difficile de faire la part des choses : pour eux, se passer de la chimie de synthèse est impensable et même absurde. Les produits phytosanitaires sont les «médicaments des plantes" pour lesquels des doses sont prescrites et respectées. Tous les agriculteurs que j'ai rencontrés affirment pratiquer une " agriculture raisonnée ", qu'ils définissent comme le fruit d'un raisonnement sur les quantités utilisées dans un "programme d'épandage" rationnel. Les produits seraient devenus tellement chers que l'on "raisonne» plus qu'autrefois. Ils incriminent la génération précédente, celle des pères qui, à les en croire, faisaient n'importe quoi. Ils s'efforcent de résoudre l'impossible équation entre d'une part le maintien des rendements et de la meilleure marge et d'autre part la protection de 
l'environnement. Cette injonction contradictoire est d'ailleurs très mal vécue par les agriculteurs. Les réglementations et les procédures sont assez tatillonnes. Elles concernent le stockage des bidons et les locaux de stockage, qui doivent respecter de nombreuses normes, le classement des produits - certains sont par exemple interdits à la vente mais utilisables dans les champs jusqu'à épuisement du stock -, les mélanges interdits et les quantités autorisées, le calendrier de l'épandage, ou encore l'utilisation « d'engrais verts »- des plantes semées entre deux cultures pour ne pas laisser le sol nu et fixer l'azote dans le sol. Ce soin apporté aux procédures est assez récent. Il date du début des années 1990, lorsque l'Union européenne a établi ses premières réglementations environnementales. Les procédures et les réglementations n'ont cessé de se multiplier. Des marchands d'équipements individuels de protection ont même vu le jour, voyant dans ces risques un véritable marché. Ainsi, chacun joue son rôle: la formatrice, les exploitants, les vendeurs d'équipement, les syndicats...

15 - SW. Dans l'ouvrage, on voit se dessiner des dissensions entre les agriculteurs, selon le syndicat auquel ils adhèrent...

16 - GE. Dans la Beauce, il n'y a que deux syndicats : la FNSEA et la Coordination rurale, la première étant très majoritaire. La Coordination rurale rassemble beaucoup d'anciens de la FNSEA, qui l'ont quittée au moment de la grande réforme libérale de la PAC en 1991-1992, car la FNSEA était incapable d'avoir à ce moment-là une position claire sur la nouvelle PAC. Ce qui n'était au début qu'une coordination est devenu rapidement un syndicat. La Coordination rurale souhaitait qu'on maintienne une PAC qui compense et qui régule le marché, c'est elle surtout qui organisa les grandes manifestations de l'époque. La FNSEA, piégée par des années de cogestion avec l'État, en était alors incapable. Beaucoup d'agriculteurs beaucerons, jusque-là protégés par l'ancienne PAC, ont été sensibles au discours de la Coordination rurale. C'était la première fois qu'une partie significative des exploitants de la plaine soutenaient une scission dans le syndicat majoritaire. Le conflit entre ces deux syndicats est donc principalement lié à l'analyse du marché, à son niveau de régulation et à la question de la protection des agriculteurs. Comparer ces deux syndicats reste néanmoins difficile : la FNSEA est pour ainsi dire un syndicat de masse - à l'échelle de la profession - présente directement ou indirectement dans tous les organismes professionnels agricoles: les Chambres d'agriculture, les SAFER (pour la gestion des terres), la MSA (pour la santé et la retraite), les caisses du Crédit agricole ou les coopératives. Du point de vue des agriculteurs qui n'y sont pas, la FNSEA fonctionne comme une "petite mafia», plus proche d'ailleurs des intérêts de l'agro-industrie que des agriculteurs. N'oublions pas que son ancien président, Xavier Beulin, était aussi un grand patron de l'industrie agroalimentaire. Autant d'éléments qui provoquent des conflits entre la base et le sommet de la FNSEA et que la Coordination rurale se fait une joie de rappeler régulièrement dans ses discours. Cependant, des points de convergence entre ces deux syndicats existent. Ils sont même nombreux : ils partagent vis-à-vis des écologistes une même colère et leur imputent une bonne part du mal-être de la profession; ils ne considèrent pas les maladies professionnels comme une réalité, et encore moins comme une question d'ordre syndical ; ils s'inscrivent dans la même logique de défense des intérêts économiques de leur profession: l'exploitation agricole est d'abord et surtout une petite entreprise qui doit dégager la meilleure marge...

17 - SW. D'autant plus qu'il y a peu de travail agricole salarié dans la Beauce, semble-t-il ? 
18 - GE. Il y a de la main-d'œuvre salariée, mais elle est saisonnière, pour les betteraves, les oignons ou les pommes de terre. Elle est composée pour l'essentiel de travailleurs des pays d'Europe centrale et orientale, des travailleurs détachés, et le recrutement passe par des groupements d'employeurs, i.e. d'exploitants, qui se mettent en lien avec des entreprises; lesquelles organisent le recrutement, le logement dans les villes qui bordent la plaine et les voyages en car jusqu'aux exploitations. Les salariés les plus stables, originaires de la région, qui les voient arriver, ont des discours très durs sur ces saisonniers des PECO. J'ai notamment eu un échange avec un salarié en CDI à plein temps, qui travaille dans un hangar à patates (qui comme souvent appartient conjointement à plusieurs chefs d'exploitation). Pour lui, comme pour d'autres, ces salariés détachés seraient des "ivrognes ", vont « se saouler » dès qu'ils touchent leur paie. Ils véhiculent de nombreux stéréotypes qui ont la vie dure.

19 - SW. Vous accordez une place de premier plan à l'Histoire: vous remontez régulièrement dans le temps, et vous adoptez une manière d'historiciser, de contextualiser, de remonter dans le temps pour montrer que cette situation a une genèse, qui ne constitue ni un cadre, ni un déterminant. Vous arrivez, en intercalant les chapitres selon leur régime d'énonciation, à faire écho à la parole des agriculteurs. Vous évitez ainsi la lourdeur de la démonstration, et pour autant, on comprend peu à peu les enjeux profondément problématiques soulevés par les propos des exploitants. Vous laissez au lecteur la liberté de tenter des corrélations, et cette discrétion donne au livre une indéniable puissance d'argumentation.

20 - GE. J'ai une formation de géographe, certes, mais ce qui m'a plu, entre autres choses, dans la géographie c'est la découverte que les paysages avaient une histoire, que beaucoup avaient été façonnés par la main de l'homme. Et que la géographie permettait de relater la genèse de cette anthropisation. Le paysage de la Beauce a une place particulière dans notre imaginaire collectif : on en trouve des photographies dans tous les manuels scolaires, même dans les livres pour enfants. C'est un paysage qui est montré comme une espèce d'évidence, avec l'idée que «c'est comme ça que l'on fait pour nourrir le monde ». Or cette plaine n'a pas toujours été ainsi. Le débat sur l'urbanisation et l'artificialisation des terres agricoles nous ferait presque oublier que la Beauce est un milieu complètement transformé par l'homme, un "paysage de synthèse » comme je l'écris dans le livre. Durant l'Antiquité la plaine était couverte de forêt. Il a fallu plusieurs siècles de déboisement pour en faire un paysage sans arbre, entièrement consacré à l'agriculture. L'entrée dans le capitalisme s'est faite au $\mathrm{XVIII}^{\mathrm{e}}$ siècle, avec le développement de la demande parisienne. A partir du XIX ${ }^{\mathrm{e}}$ siècle, c'est l'entrée dans la modernité technicienne et le rapprochement progressif des exploitations agricoles du monde de l'industrie - les intrants de synthèse et les premières machines en amont, et les usines agroalimentaires en aval. Sur ce plan, les deux guerres mondiales ont joué un rôle d'accélérateur. La Première Guerre mondiale et la saignée démographique ont entraîné un premier grand mouvement de concentration foncière des terres. Au sortir de la guerre, les parcelles appartiennent à de moins en moins de personnes. La Seconde Guerre mondiale a eu au moins trois effets, liées entre eux. Sur les semences d'une part: c'est sous Vichy que l'on a commencé à « contrôler le vivant » (Bonneuil, 2013, op. cit.), à chercher la pureté dans la semence et à conformer les grains aux attentes de l'industrie, qu'elle soit en aval, avec les industries agroalimentaires, ou en amont (les machines exigeant par exemple que le blé ne soit pas trop irrégulier, ni trop haut ni trop bas). Sur l'utilisation de la 
chimie de synthèse d'autre part: la guerre a produit des surplus qu'on écoule sous forme de produits phytosanitaires (Fressoz, op. cit.) pour protéger des semences, sélectionnées pour leur rendement mais de plus en plus fragiles. Sur la mécanisation, enfin : dès 1947 en France, avec le plan Marshall, il s'agissait d'écouler les machines américaines, avec un esprit atlantiste qui entendait développer les pays d'Europe de l'Ouest pour éviter qu'ils ne votent communiste. Et pour conformer l'espace à ces innovations, pour laisser de la place aux machines et faire des économies d'échelle, on redessina le tracé des parcelles par des remembrements successifs. L'après-guerre est un moment de "recadrage modernisateur", dirait François Jarrige (op. cit.), un moment où l'idée devient dominante de moderniser les campagnes. Et la Beauce fut, à chaque fois, l'une des premières régions à introduire ces innovations, à mettre en œuvre ces grandes transformations. - SW. Quelle était la coloration politique d'une campagne comme la Beauce?

-GE. C'est le travail de Jean-Claude Farcy (1989) qui m'a été très utile. Depuis le $\mathrm{XIX}^{\mathrm{e}}$ siècle, la structure sociale de la Beauce n'a jamais donné de mouvement social : on $\mathrm{y}$ a vécu longtemps assez bien avec de petites parcelles qui permettaient de dégager un revenu. Beaucoup de salariés de grands domaines avaient, en parallèle, leur petite exploitation, il y avait un équilibre social. Entre le XIX ${ }^{e}$ siècle et la Première Guerre mondiale, il n'y a pas eu de prolétarisation des salariés agricoles. Les discours ouvriers prenaient peu, d'autant moins que les paysans avaient tous cet espoir de s'établir euxmêmes et d'acheter une exploitation, ce qui est le cas de la plupart des grands-parents des exploitants que j'ai rencontrés. En 1936, il n'y a pas eu de grands mouvements, sauf peut-être à Châteaudun, où il y a eu quelques journées de grève, car il y avait dans le Dunois quelques syndicalistes ouvriers qui ont su s'adresser aux salariés agricoles. À part cet épisode marginal, ce sont des terres qui votent à droite depuis longtemps, même s'ils sont historiquement partagés entre catholiques et républicains. On trouve dans le mouvement des coopératives agricoles des coopératives tenues par le mouvement chrétien et d'autres par le mouvement républicain: les «bleus » et les " rouges », c'est une opposition très prégnante dans la mémoire collective locale.

- SW. Et la question du célibat agricole, l'avez-vous rencontrée?

24 - GE. Il y a des absents dans ce livre. Les femmes sont absentes. Comment parler des absentes? Les agriculteurs que j'ai rencontrés sont tous des hommes, mais ne sont pas tous célibataires. Une moitié d'entre eux environ ne l'est pas. Il y a des chefs d'exploitation qui sont en couple, avec des enfants. Dans ces cas, les femmes travaillent systématiquement en dehors des exploitations et en dehors de la plaine (dans les villes, à Chartres, à Orléans, etc.). J'en ai croisé, mais elles ne sont pas dans le monde de l'agriculture, ce n'est pas leur univers professionnel. Il y a aussi beaucoup de femmes parmi les salariés saisonniers (qu'elles viennent des villes alentours ou des PECO) notamment pour la saison des patates ou des betteraves. Encore une fois, il est difficile de les rencontrer, à moins d'être sur place précisément au moment de la récolte. $\mathrm{Au}$ salon professionnel «Innov'agri », à Outarville où je me suis rendu, et qui fait l'objet d'un chapitre dans le livre, c'est un univers masculin que j'ai rencontré, à l'exception des personnes qui tenaient les stands et s'occupaient de l'aspect commercial. Les chefs d'exploitation étaient tous des hommes.

25 - SW. La question de la reprise se pose comme ailleurs, j'imagine?

26 - GE. Elle revient souvent. Tous les agriculteurs qui travaillent dans la Beauce ont hérité, on ne trouve aucun «Hors cadre familial» (HCF), terme qui désigne ceux qui 
s'installeraient en venant d'ailleurs. Aujourd'hui, à ce stade de l'histoire de la plaine, ceux qui reprennent ont tous hérité. C'est important de garder cet élément à l'esprit : pour la plupart des exploitants que j'ai rencontrés, il y a une sorte d'impératif moral à reprendre, il faut qu'il y en ait un dans la famille qui reprenne. Ceux qui sont partis, de même, je ne les ai pas rencontrés, ce sont d'autres absents. Pour ceux qui restent, il y a comme une obligation à «faire tourner la boutique » sous peine de devoir vendre à ses voisins, ce qui serait un « crève-cœur ", d'autant que les relations ne sont pas toujours pacifiées. Le premier agriculteur dont je propose le portrait dans le livre, Michel, présente cet agrandissement comme tragique.

- SW. Avez-vous l'impression que les investisseurs extérieurs sont présents?

28 - GE. Il y en a, mais finalement c'est assez rare dans la Beauce, à la différence du Bordelais, de la Champagne ou des Landes. Le fait existe, mais reste minoritaire, il y a suffisamment d'exploitants qui cherchent à s'agrandir et qui ont les moyens d'acheter l'exploitation du voisin. On ne peut pas comprendre la logique de la Beauce si on ne comprend pas que beaucoup se donnent pour mission de faire vivre l'entreprise des parents et grands-parents. Le dernier des agriculteurs à apparaître dans le livre, Cédric, dit bien qu'il ne se destinait pas à devenir agriculteur, il avait commencé une carrière de professeur de mathématiques. Son père avait failli " couler l'affaire », ce qui a décidé son grand-père à lui demander de "reprendre ». Ce poids de l'héritage s'ajoute à l'impératif de survie économique: on ne va pas «faire le mariole» et tenter des alternatives bio, alors même que, sur le principe, la plupart des agriculteurs ne seraient pas contre. C'est justement cela qui est intéressant : avec un raisonnement économique, ils s'adapteraient au marché comme ils le font pour les toutes leurs productions. Si on leur démontrait que le bio est viable économiquement, ils l'adopteraient. Pour l'instant, pour eux, le bio reste un marché de niche, ce n'est pas cela qui va leur permettre de faire marcher leur exploitation.

29 - SW. Finalement, ce titre "plaine ", peut surprendre pour un des plateaux les plus emblématiques du Bassin parisien...

30 - GE. «La plaine ", c'est le mot qu'on emploie localement pour désigner la Beauce. Ce nom revient tout le temps parmi les professionnels de la Beauce alors que ce n'est pas une plaine. Dans ce terme de "plaine ", il y a tout l'imaginaire de la conquête, de la plaine fertile, de l'occupation et de la mise en valeur, qui renvoie à tout un imaginaire en géographie, celui de l'action humaine et celui des possibles.

\section{BIBLIOGRAPHY}

Allain M. (dir.), 1924. Géographie universelle illustrée Quillet (Physique-Economique-Humaine). Paris, Quillet, 4 tomes.

Bonneuil C., Thomas F., Petitjean O., 2012. Semences, une histoire politique. Amélioration des plantes, agriculture et alimentation en France depuis la seconde Guerre mondiale. Paris, Fondation Charles Léopold Mayer, 2016 p. 
Bonneuil C., 2013. Comment l'agriculture est entrée dans la logique productiviste. In Larqué L., Pestre D. (dir.), Les sciences, ça nous regarde. Paris, Les Empêcheurs de penser en rond.

Charbonneau B., 1973. Tristes campagnes. Essai. La mise à sac du sud-ouest. Paris, Denoël, 239 p.

Claudel P., 1946. L'œil écoute. Paris, Gallimard, 246 p.

Deffontaines P., Jean-Brunhes Delamarre M., 1960. Géographie universelle Larousse. Paris, Larousse, 3 volumes, 420 p. +389 p. +407 p.

Dejours C., 1998. Souffrance en France. La banalisation de l'injustice sociale. Paris, Le Seuil, 208 p.

Elie G., 2016. L'école en Seine-Saint-Denis : une pauvre école pour des enfants de pauvres? Hérodote, vol. 162, no 3, p. 85-98.

Elie G., Foscolo E., 2007. L'instrumentalisation de l'espace. Rendre visible un problème économique et social pour alerter le politique. EchoGéo [En ligne], 1 | 2007, mis en ligne le 17 mars 2008. http://journals.openedition.org/echogeo/992 ; DOI : 10.4000/echogeo.992

Elie G., Popelard A., Vannier P., 2009. William Bunge, le géographe révolutionnaire de Détroit. Le Monde diplomatique, Visions cartographiques, 29 décembre, https://blog.mondediplo.net/ 2009-12-29-William-Bunge-le-geographe-revolutionnaire-de

Elie G., Popelard A., Vannier P., 2010. Exode urbain, exil rural. Des pauvres relégués à la campagne. Le Monde diplomatique, $\mathrm{n}^{\circ}$ 677, p. 1-10.

Elie G., Popelard A., Vannier P., 2013. Le laboratoire grec : quand la crise défait le territoire. Le Monde diplomatique, vol. 707, no 2, p. 8-9.

Farcy J.-C., 1989. Les paysans beaucerons au XIX siècle. Chartres, Société archéologique d'Eure-etLoir, 2 volumes, $1229 \mathrm{p}$.

Fressoz J.-B., 2012. L'Apocalypse joyeuse. Une histoire du risque technologique. Paris, Le Seuil, 320 p. Géographie universelle illustrée quillet, 1924

Jarrige F., 2009. Face au monstre mécanique. Une histoire des résistances à la technique. Paris, IMHO, $169 \mathrm{p}$.

Jas N., 2005. Déqualifier le paysan, introniser l'agronome. France, 1850-1914. Ecologie et politique, $\mathrm{n}^{\circ} 31$, p. $45-55$.

Linhart R., 1978. L'Établi. Paris, Minuit, 178 p.

Linhart R., 1981. Le Sucre et la Faim. Enquête dans les régions sucrières du nord-est brésilien. Paris, Minuit, $96 \mathrm{p}$.

\section{AUTHORS}

\section{GATIEN ELIE}

Gatien Elie, gatien.elie@gmail.com, est géographe, professeur agrégé au lycée Suger de SaintDenis. Il a publié :

- Elie G., 2018. La Plaine. Récits de travailleurs du productivisme agricole. Paris, Editions Amsterdam, Collection L'Ordinaire du capital, $154 \mathrm{p}$. 


\section{SERGE WEBER}

Serge Weber, serge.weber@u-pem.fr, est Professeur d'université en géographie à l'UPEM

(Université Paris-Est Marne-la-Vallée) et membre du laboratoire ACP. 